\title{
Myasthenia Gravis
}

National Cancer Institute

\section{Source}

National Cancer Institute. Myasthenia Gravis. NCI Thesaurus. Code C60989.

A chronic autoimmune neuromuscular disorder characterized by skeletal muscle weakness. It is caused by the blockage of the acetylcholine receptors at the neuromuscular junction. 